\title{
Common Fixed Point Theorem of Comatible Mappings in Metric Space
}

\author{
R K Gujetiya', Mala Hakwadiya,", Dheeraj Kumari Mali \\ ${ }^{1}$ Department of Mathematics, Govt. P. G. College, Neemuch, India \\ ${ }^{2}$ Pacific Academy of Higher Education, and Research University Udaipur, Rajasthan, India \\ *Corresponding author: manuvi9the@gmail.com
}

Received June 23, 2014; Revised July 19, 2014; Accepted July 24, 2014

\begin{abstract}
In this paper we prove a common fixed point theorem of compatible mappings of type (R). Our result modify the result of M. Koireng Meitei [4].
\end{abstract}

Keywords: fixed point, complete metric space, compatible mappings

Cite This Article: R K Gujetiya, Mala Hakwadiya, and Dheeraj Kumari Mali, "Common Fixed Point Theorem of Comatible Mappings in Metric Space." American Journal of Applied Mathematics and Statistics, vol. 2, no. 4 (2014): 207-211. doi: 10.12691/ajams-2-4-5.

\section{Introduction}

The first important result in the theory of fixed point of compatible mappings was obtained by Gerald Jugck in 1986 [2] as a generalization of commuting mappings. Pathak, Chang and Cho [3] in 1994 introduced the concept of compatible mappings of type(P). In 2004 Rohen, Singh and shambhu [5] introduced the concept of compatible mappings of type(R) by combining the definitions of compatible mappings and compatible mappings of type $(\mathrm{P})$. The aim of this paper is to prove a common fixed point theorem of compatible mappings of type(R) in metric space by considering eight self mappings.

\section{Preliminaries}

Definition 2.1: [2] A metric space is given by a set $X$ and a distance function $\mathrm{d}: \mathrm{X} \times \mathrm{X} \rightarrow \mathbb{R}$ such that

(i) (Positivity) For all $x, y \in X, 0 \leq d(x, y)$.

(ii) (Non-degenerated) For all $\mathrm{x}, \mathrm{y} \in \mathrm{X}$,

$$
0=\mathrm{d}(\mathrm{x}, \mathrm{y}) \Leftrightarrow \mathrm{x}=\mathrm{y} .
$$

(iii) (Symmetry) For all $\mathrm{x}, \mathrm{y} \in \mathrm{X}$,

$$
\mathrm{d}(\mathrm{x}, \mathrm{y})=\mathrm{d}(\mathrm{y}, \mathrm{x})
$$

(iv) (Triangle inequality) For all $\mathrm{x}, \mathrm{y}, \mathrm{z} \in \mathrm{X}$,

$$
d(x, y) \leq d(x, z)+d(z, y) .
$$

Definition 2.2: [4] Let $S$ and $T$ be mappings from a complete metric space $\mathrm{X}$ into itself. The mappings $\mathrm{S}$ and $\mathrm{T}$ are said to be compatible if $\lim _{n \rightarrow \infty} d\left(\operatorname{STx}_{n}, \mathrm{TSx}_{\mathrm{n}}\right)=0$ whenever $\left\{x_{n}\right\}$ is a sequence in $X$ such that $\lim _{n \rightarrow \infty} T x_{n}=\lim _{n \rightarrow \infty} S x_{n}=t$ for some $t \in X$.
Definition 2.3: [4] Let $S$ and $T$ be mappings from a complete metric space $\mathrm{X}$ into itself. The mappings $\mathrm{S}$ and $\mathrm{T}$ are said to be compatible of type (P) if $\lim _{n \rightarrow \infty} d\left(S x_{n}, T T x_{n}\right)=0$ whenever $\left\{x_{n}\right\}$ is a sequence in $\mathrm{X}$ such that for $\lim _{\mathrm{n} \rightarrow \infty} \mathrm{Sx}_{\mathrm{n}}=\lim _{\mathrm{n} \rightarrow \infty} \mathrm{Tx}_{\mathrm{n}}=\mathrm{t}$ for some $\mathrm{t} \in \mathrm{X}$.

Definition 2.4: [4] Let $S$ and $T$ be mappings from a complete metric space $\mathrm{X}$ into itself. The mappings $\mathrm{S}$ and $\mathrm{T}$ are said to be compatible of type (R) if $\lim _{n \rightarrow \infty} d\left(\operatorname{STx}_{n}, \operatorname{TSx}_{n}\right)=0$ and $\lim _{n \rightarrow \infty} d\left(\operatorname{SSx}_{n}, \operatorname{TTx}_{n}\right)=0$ whenever $\left\{x_{n}\right\}$ is a sequence in $X$ such that for $\lim _{\mathrm{n} \rightarrow \infty} S x_{n}=\lim _{\mathrm{n} \rightarrow \infty} T x_{n}=t$ for some $\mathrm{t} \in \mathrm{X}$.

Proposition 2.5. [4] Let $S$ and $T$ be mappings from a complete metric space $(X, d)$ into itself. If a pair $\{S, T\}$ is compatible of type $(\mathrm{R})$ on $\mathrm{X}$ and $\mathrm{Sz}=\mathrm{Tz}$ for $\mathrm{z} \in \mathrm{X}$, Then $\mathrm{STz}=\mathrm{TSz}=\mathrm{SSz}=\mathrm{TTz}$.

Proposition 2.6. [4] Let $S$ and $T$ be mappings from a complete metric space (X, d) into itself. If a pair $\{\mathrm{S}, \mathrm{T}\}$ is compatible of type (R) on $\mathrm{X}$ and $\lim _{n \rightarrow \infty} S x_{n}=\lim _{n \rightarrow \infty} T x_{n}=z$ for some $z \in X$, then we have

(i) $\mathrm{d}\left(\mathrm{TSx}_{\mathrm{n}}, \mathrm{Sz}\right) \rightarrow 0$ as $\mathrm{n} \rightarrow \infty$ if $\mathrm{S}$ is continuous,

(ii) $\mathrm{d}\left(\mathrm{STx}_{\mathrm{n}}, \mathrm{Tz}\right) \rightarrow 0$ as $\mathrm{n} \rightarrow \infty$ if $\mathrm{T}$ is continuous and

(iii) $\mathrm{STz}=\mathrm{TSz}$ and $\mathrm{Sz}=\mathrm{Tz}$ if $\mathrm{S}$ and $\mathrm{T}$ are continuous at $\mathrm{z}$. Lemma 2.7. [4] Let $A, B, S$ and $T$ be mapping from $a$ metric space $(X, d)$ into itself satisfying the following conditions:

(1) $\mathrm{A}(\mathrm{X}) \subseteq \mathrm{T}(\mathrm{X})$ and $\mathrm{B}(\mathrm{X}) \subseteq \mathrm{S}(\mathrm{X})$

$$
\begin{aligned}
{[\mathrm{d}(\text { Ax }, \text { By })]^{2} \leq } & \mathrm{k}_{1}\left[\begin{array}{l}
\mathrm{d}(\text { Ax }, \text { Sx }) \mathrm{d}(\text { By, Ty }) \\
+\mathrm{d}(\text { By, Sx }) \mathrm{d}(\text { Ax }, \text { Ty })
\end{array}\right] \\
& +\mathrm{k}_{2}\left[\begin{array}{l}
\mathrm{d}(\text { Ax }, \text { Sx }) \mathrm{d}(\text { Ax }, \text { Ty }) \\
+\mathrm{d}(\text { By, Ty }) \mathrm{d}(\text { By, Sx })
\end{array}\right]
\end{aligned}
$$

Where $0 \leq \mathrm{k}_{1}+2 \mathrm{k}_{2}<1 ; \mathrm{k}_{1}, \mathrm{k}_{2} \geq 0$. 
(3) Let $x_{0} \in X$ then by (1) there exists $x_{1} \in X$ such that $\mathrm{Tx}_{1}=\mathrm{Ax}_{0}$ and for $\mathrm{x}_{1}$ there exists $\mathrm{x}_{2} \in \mathrm{X}$ such that $\mathrm{Sx}_{2}=\mathrm{Bx}_{1}$ and so on. Continuing this process we can define a sequence $\left\{y_{n}\right\}$ in $X$ such that

$$
\begin{aligned}
& \mathrm{y}_{2 \mathrm{n}+1}=\mathrm{Tx}_{2 \mathrm{n}+1}=A x_{2 \mathrm{n}} \\
& \text { and } \mathrm{y}_{2 \mathrm{n}}=\mathrm{Sx}_{2 \mathrm{n}}=B x_{2 \mathrm{n}-1}
\end{aligned}
$$

Then the sequence $\left\{y_{n}\right\}$ is Cauchy sequence in $X$.

Theorem: [4] Let A, B, S and $\mathrm{T}$ be mapping from a metric space $(X, d)$ into itself satisfying the following conditions:

(1) $\mathrm{A}(\mathrm{X}) \subseteq \mathrm{T}(\mathrm{X})$ and $\mathrm{B}(\mathrm{X}) \subseteq \mathrm{S}(\mathrm{X})$

$$
\begin{aligned}
{[\mathrm{d}(\text { Ax, By })]^{2} \leq } & \mathrm{k}_{1}\left[\begin{array}{l}
\mathrm{d}(\text { Ax }, \text { Sx }) \mathrm{d}(\text { By, Ty }) \\
+\mathrm{d}(\text { By, Sx }) \mathrm{d}(\text { Ax }, \text { Ty })
\end{array}\right] \\
& +\mathrm{k}_{2}\left[\begin{array}{l}
\mathrm{d}(\text { Ax }, \text { Sx }) \mathrm{d}(\text { Ax }, \text { Ty }) \\
+\mathrm{d}(\text { By, Ty }) \mathrm{d}(\text { By }, \text { Sx })
\end{array}\right]
\end{aligned}
$$

Where $0 \leq \mathrm{k}_{1}+2 \mathrm{k}_{2}<1 ; \mathrm{k}_{1}, \mathrm{k}_{2} \geq 0$.

(3) Let $x_{0} \in X$ then by (1) there exists $x_{1} \in X$ such that $\mathrm{Tx}_{1}=\mathrm{Ax}_{0}$ and for $\mathrm{x}_{1}$ there exists $\mathrm{x}_{2} \in \mathrm{X}$ such that $\mathrm{Sx}_{2}=\mathrm{Bx}_{1}$ and so on. Continuing this process we can define a sequence $\left\{y_{n}\right\}$ in $X$ such that

$$
\begin{aligned}
& \mathrm{y}_{2 \mathrm{n}+1}=\mathrm{Tx}_{2 \mathrm{n}+1}=\mathrm{Ax}_{2 \mathrm{n}} \\
& \text { and } \mathrm{y}_{2 \mathrm{n}}=\mathrm{Sx}_{2 \mathrm{n}}=\mathrm{Bx}_{2 \mathrm{n}-1} .
\end{aligned}
$$

Then the sequence $\left\{y_{n}\right\}$ is Cauchy sequence in $X$.

(4) One of A, B, S or T is continuous.

(5) $[A, S]$ and $[B, T]$ are compatible of type (R) on X. Then $A, B, S$ and $T$ have a unique common fixed point in $\mathrm{X}$.

\section{Main Result}

Lemma 3.1: Let C, D, E, F, K, M, P and V be self maps of a complete metric space $(\mathrm{X}, \mathrm{d})$ satisfying the following conditions:

(1) $\mathrm{C}(\mathrm{X}) \subseteq \mathrm{DPV}(\mathrm{X})$ and $\mathrm{E}(\mathrm{X}) \subseteq \mathrm{FKM}(\mathrm{X})$

(2)

$$
\begin{aligned}
{[\mathrm{d}(\text { Cx }, \text { Ey })]^{2} \leq } & \alpha_{1}\left[\begin{array}{l}
\mathrm{d}(\text { Cx }, \text { FKMx }) \mathrm{d}(\text { Ey, DPVy }) \\
+\mathrm{d}(\text { Ey, FKMx }) \mathrm{d}(\text { Cx }, \text { DPVy })
\end{array}\right] \\
& +\alpha_{2}\left[\begin{array}{l}
\mathrm{d}(\text { Cx }, \text { FKMx }) \mathrm{d}(\text { Cx }, \text { DPVy }) \\
+\mathrm{d}(\text { Ey, DPVy }) \mathrm{d}(\text { Ey, FKMx })
\end{array}\right]
\end{aligned}
$$

Where $0 \leq \alpha_{1}+2 \alpha_{2}<1 ; \propto_{1}, \propto_{2} \geq 0$.

(3) Let $x_{0} \in X$ then by (1) there exists $x_{1} \in X$ such that $\mathrm{DPVx}_{1}=\mathrm{Cx}_{0}$ and for $\mathrm{x}_{1}$ there exists $\mathrm{x}_{2} \in \mathrm{X}$ such that $\mathrm{FKMx}_{2}=\mathrm{Ex}_{1}$ and so on.continuing this process we candefine a sequence $\left\{\mathrm{y}_{\mathrm{n}}\right\}$ in $\mathrm{X}$ such that

$$
\begin{aligned}
& \mathrm{y}_{2 \mathrm{n}+1}=\mathrm{DPVx}_{2 \mathrm{n}+1}=\mathrm{Cx}_{2 \mathrm{n}} \\
& \text { and } \mathrm{y}_{2 \mathrm{n}}=\mathrm{FKMx}_{2 \mathrm{n}}=\mathrm{Ex}_{2 \mathrm{n}-1}
\end{aligned}
$$

Then the sequence $\left\{y_{n}\right\}$ is a Cauchy sequence in $X$.

Proof: By condition (2) and (3), we have

$$
\begin{aligned}
& {\left[d\left(y_{2 n+1}, y_{2 n}\right)\right]^{2}=\left[d\left(C_{2 n}, E x_{2 n-1}\right)\right]^{2}} \\
& \leq \alpha_{1}\left[\begin{array}{l}
\mathrm{d}\left(\mathrm{Cx}_{2 \mathrm{n}}, \mathrm{FKMx}_{2 \mathrm{n}}\right) \mathrm{d}\left(\mathrm{Ex}_{2 \mathrm{n}-1}, \mathrm{DPVx}_{2 \mathrm{n}-1}\right) \\
++\mathrm{d}\left(\mathrm{Ex}_{2 \mathrm{n}-1}, \mathrm{FKMx}_{2 \mathrm{n}}\right) \mathrm{d}\left(\mathrm{Cx}_{2 \mathrm{n}}, \mathrm{DPVx}_{2 \mathrm{n}-1}\right)
\end{array}\right] \\
& +\alpha_{2}\left[\begin{array}{l}
\mathrm{d}\left(\mathrm{Cx}_{2 \mathrm{n}}, \mathrm{FKMx}_{2 \mathrm{n}}\right) \mathrm{d}\left(\mathrm{Cx}_{2 \mathrm{n}}, \mathrm{DPVx}_{2 \mathrm{n}-1}\right) \\
+\mathrm{d}\left(\mathrm{Ex}_{2 \mathrm{n}-1}, \mathrm{DPVx}_{2 \mathrm{n}-1}\right) \mathrm{d}\left(\mathrm{Ex}_{2 \mathrm{n}-1}, \mathrm{FKMx}_{2 \mathrm{n}}\right)
\end{array}\right] \\
& =\alpha_{1}\left[d\left(y_{2 n+1}, y_{2 n}\right) d\left(y_{2 n}, y_{2 n-1}\right)+0\right] \\
& +\alpha_{2}\left[d\left(y_{2 n+1}, y_{2 n}\right) d\left(y_{2 n+1}, y_{2 n-1}\right)+0\right] \\
& {\left[d\left(y_{2 n+1}, y_{2 n}\right)\right] \leq \alpha_{1} d\left(y_{2 n}, y_{2 n-1}\right)+\alpha_{2}\left[\begin{array}{l}
d\left(y_{2 n+1}, y_{2 n}\right) \\
+d\left(y_{2 n}, y_{2 n-1}\right)
\end{array}\right]} \\
& {\left[\mathrm{d}\left(\mathrm{y}_{2 \mathrm{n}+1}, \mathrm{y}_{2 \mathrm{n}}\right)\right] \leq \operatorname{pd}\left(\mathrm{y}_{2 \mathrm{n}}, \mathrm{y}_{2 \mathrm{n}-1}\right)} \\
& \text { where } \mathrm{p}=\frac{\alpha_{1}+\alpha_{2}}{1-\alpha_{2}}<1 \text {. }
\end{aligned}
$$

Hence $\left\{y_{n}\right\}$ is Cauchy sequence.

Theorem 3.2: Let C, D, E, F, K, M, P and V be self maps of a complete metric space $(\mathrm{X}, \mathrm{d})$ satisfying the following conditions:

(1) $\mathrm{C}(\mathrm{X}) \subseteq \mathrm{DPV}(\mathrm{X})$ and $\mathrm{E}(\mathrm{X}) \subseteq \mathrm{FKM}(\mathrm{X})$

(2)

$$
\begin{aligned}
{[\mathrm{d}(\text { Cx }, \text { Ey })]^{2} \leq } & \alpha_{1}\left[\begin{array}{l}
\mathrm{d}(\text { Cx }, \text { FKMx }) \mathrm{d}(\text { Ey, DPVy }) \\
+\mathrm{d}(\text { Ey, FKMx }) \mathrm{d}(\text { Cx }, \text { DPVy })
\end{array}\right] \\
& +\alpha_{2}\left[\begin{array}{l}
\mathrm{d}(\text { Cx, FKMx }) \mathrm{d}(\text { Cx }, \text { DPVy }) \\
+\mathrm{d}(\text { Ey, DPVy }) \mathrm{d}(\text { Ey, FKMx })
\end{array}\right]
\end{aligned}
$$

Where $0 \leq \alpha_{1}+2 \alpha_{2}<1 ; \propto_{1}, \propto_{2} \geq 0$.

(3) Let $x_{0} \in X$ then by (1) there exists $x_{1} \in X$ such that $\mathrm{DPVx}_{1}=\mathrm{Cx}_{0}$ and for $\mathrm{x}_{1}$ there exists $\mathrm{x}_{2} \in \mathrm{X}$ such that $\mathrm{FKMx}_{2}=\mathrm{Ex}_{1}$ and so on.continuing this process we candefine a sequence $\left\{\mathrm{y}_{\mathrm{n}}\right\}$ in $\mathrm{X}$ such that

$\mathrm{y}_{2 \mathrm{n}+1}=D P V \mathrm{x}_{2 \mathrm{n}+1}=\mathrm{Cx}_{2 \mathrm{n}}$ and $\mathrm{y}_{2 \mathrm{n}}=\mathrm{FKMx}_{2 \mathrm{n}}=\mathrm{Ex}_{2 \mathrm{n}-1}$.

Then the sequence $\left\{y_{n}\right\}$ is a Cauchy sequence in $X$.

(4) One of C, E, FKM, DPV is continuous.

(5) $[\mathrm{C}, \mathrm{FKM}]$ and $[\mathrm{E}, \mathrm{DPV}]$ are compatible of type (R) on $\mathrm{X}$.

Then C, D, E, F, K, M, P and V have a unique common fixed point in $\mathrm{X}$.

Proof: By lemma 3.1, $\left\{\mathrm{y}_{\mathrm{n}}\right\}$ is Cauchy sequence. and since $\mathrm{X}$ is complete so there exists a point $\mathrm{z} \in \mathrm{X}$ such that $\lim \mathrm{y}_{\mathrm{n}}=\mathrm{z}$ as $\mathrm{n} \rightarrow \infty$.

Consequently subsequences $\mathrm{Cx}_{2 \mathrm{n}}, \mathrm{FKMx}_{2 \mathrm{n}}, \mathrm{Ex}_{2 \mathrm{n}-1}$ and $\mathrm{DPVx}_{2 \mathrm{n}+1}$ converges to $\mathrm{z}$. Let FKM be continuous. Since $\mathrm{C}$ and FKM are compatible of type (R) on $\mathrm{X}$. Then by proposition 2.6, We have (FKM) ${ }^{2} \mathrm{x}_{2 \mathrm{n}} \rightarrow \mathrm{FKMz}$ and $(\mathrm{C})(\mathrm{FKM}) \mathrm{x}_{2 \mathrm{n}} \rightarrow \mathrm{FKMz}$ as $\mathrm{n} \rightarrow \infty$.

Now by condition (2), we have $\left[\mathrm{d}\left(\mathrm{CFKMx}_{2 \mathrm{n}}, \mathrm{Ex}_{2 \mathrm{n}-1}\right)\right]$

$\leq \alpha_{1}\left[\begin{array}{l}\mathrm{d}\left(\mathrm{CFKMx}_{2 \mathrm{n}},(F K M)^{2} \mathrm{x}_{2 \mathrm{n}}\right) \mathrm{d}\left(\mathrm{Ex}_{2 \mathrm{n}-1}, \mathrm{DPVx}_{2 \mathrm{n}-1}\right) \\ +\mathrm{d}\left(\mathrm{Ex}_{2 \mathrm{n}-1},(F K M)^{2} \mathrm{x}_{2 \mathrm{n}}\right) \mathrm{d}\left(\mathrm{CFKMx}_{2 \mathrm{n}}, \operatorname{DPVx}_{2 \mathrm{n}-1}\right)\end{array}\right]$ $+\alpha_{2}\left[\begin{array}{l}\mathrm{d}\left(\mathrm{CFKMx}_{2 \mathrm{n}},(F K M)^{2} \mathrm{x}_{2 \mathrm{n}}\right) \mathrm{d}\left(\mathrm{CFKMx}_{2 \mathrm{n}}, \operatorname{DPVx}_{2 \mathrm{n}-1}\right) \\ +\mathrm{d}\left(\mathrm{Ex}_{2 \mathrm{n}-1}, \operatorname{DPVx}_{2 \mathrm{n}-1}\right) \mathrm{d}\left(\mathrm{Ex}_{2 \mathrm{n}-1},(F K M)^{2} x_{2 n}\right)\end{array}\right]$ 


$$
\begin{aligned}
& {\left[\mathrm{d}\left(\mathrm{CFKMx}_{2 \mathrm{n}}, \mathrm{y}_{2 \mathrm{n}-1}\right)\right]^{2}} \\
& \leq \alpha_{1}\left[\begin{array}{l}
d\left(\operatorname{CFKMx}_{2 n},(F K M)^{2} x_{2 n}\right) d\left(y_{2 n}, y_{2 n-1}\right) \\
+d\left(y_{2 n},(F K M)^{2} x_{2 n}\right) d\left(C_{C K M x} x_{2 n-1}, y_{2 n-1}\right)
\end{array}\right] \\
& +\alpha_{2}\left[\begin{array}{l}
d\left(C_{C K M x_{2 n}},(F K M)^{2} x_{2 n}\right) d\left(C_{\left.C K M x_{2 n}, y_{2 n-1}\right)}\right) \\
+d\left(y_{2 n}, y_{2 n-1}\right) d\left(y_{2 n},(F K M)^{2} x_{2 n}\right)
\end{array}\right]
\end{aligned}
$$

Letting $\mathrm{n} \rightarrow \infty$, we have

$$
\begin{aligned}
& {[\mathrm{D}(\text { FKMz, z })]^{2} \leq \alpha_{1}\left[\begin{array}{l}
\mathrm{d}(\text { FKMz, FKMz }) \mathrm{d}(\mathrm{z}, \mathrm{z}) \\
+\mathrm{d}(\mathrm{z}, \text { FKMz }) \mathrm{d}(\text { FKMz, z })
\end{array}\right]} \\
& +\alpha_{2}\left[\begin{array}{l}
\mathrm{d}(\text { FKMz, FKMz }) \mathrm{d}(\text { FKMz, z }) \\
+\mathrm{d}(\mathrm{z}, \mathrm{z}) \mathrm{d}(\mathrm{z}, \text { FKMz })
\end{array}\right] \\
& {[\mathrm{d}(\text { FKMz, z })]^{2} \leq \alpha_{1}[\mathrm{~d}(\mathrm{z}, \text { FKMz }) \mathrm{d}(\text { FKMz }, \mathrm{z})]} \\
& {[\mathrm{d}(\mathrm{FKMz}, \mathrm{z})]^{2} \leq \alpha_{1}[\mathrm{~d}(\mathrm{FKMz}, \mathrm{z})]^{2}}
\end{aligned}
$$

Which is a contradiction. Hence

$$
\mathrm{FKMz}=\mathrm{z}
$$

Now by putting $\mathrm{x}=\mathrm{z}$ and $\mathrm{y}=\mathrm{x}_{2 \mathrm{n}-1}$ in condition (2), then we have

$$
\begin{aligned}
& {\left[\mathrm{d}\left(\mathrm{Cz}, \mathrm{Ex}_{2 \mathrm{n}-1}\right)\right]^{2}} \\
& \leq \alpha_{1}\left[\begin{array}{l}
\mathrm{d}(\mathrm{Cz}, \mathrm{FKMz}) \mathrm{d}\left(\mathrm{Ex}_{2 \mathrm{n}-1}, \mathrm{DPVx}_{2 \mathrm{n}-1}\right) \\
+\mathrm{d}\left(\mathrm{Ex}_{2 \mathrm{n}-1}, \mathrm{FKMz}\right) \mathrm{d}\left(\mathrm{Cz}, \mathrm{DPVx}_{2 \mathrm{n}-1}\right)
\end{array}\right] \\
& +\alpha_{2}\left[\begin{array}{l}
\mathrm{d}(\mathrm{Cz}, \mathrm{FKMz}) \mathrm{d}\left(\mathrm{Cz}, \mathrm{DPVx}_{2 \mathrm{n}-1}\right) \\
+\mathrm{d}\left(\mathrm{Ex}_{2 \mathrm{n}-1}, \mathrm{DPVx}_{2 \mathrm{n}-1}\right) \mathrm{d}\left(\mathrm{Ex}_{2 \mathrm{n}-1}, \mathrm{FKMz}\right)
\end{array}\right] \\
& {\left[\mathrm{d}\left(\mathrm{Cz}, \mathrm{y}_{2 \mathrm{n}}\right)\right]^{2}} \\
& \leq \alpha_{1}\left[\begin{array}{l}
d(C z, F K M z) d\left(y_{2 n}, y_{2 n-1}\right) \\
+d\left(y_{2 n}, F K M z\right) d\left(C z, y_{2 n-1}\right)
\end{array}\right] \\
& +\alpha_{2}\left[\begin{array}{l}
\mathrm{d}(\mathrm{Cz}, \mathrm{FKMz}) \mathrm{d}\left(\mathrm{Cz}, \mathrm{y}_{2 \mathrm{n}-1}\right) \\
+\mathrm{d}\left(\mathrm{y}_{2 \mathrm{n}}, \mathrm{y}_{2 \mathrm{n}-1}\right) \mathrm{d}\left(\mathrm{y}_{2 \mathrm{n}}, \mathrm{FKMz}\right)
\end{array}\right]
\end{aligned}
$$

Letting $\mathrm{n} \rightarrow \infty$, we have

$$
\begin{aligned}
{[\mathrm{d}(\mathrm{Cz}, \mathrm{z})]^{2} \leq } & \alpha_{1}\left[\begin{array}{l}
\mathrm{d}(\mathrm{Cz}, \mathrm{z}) \mathrm{d}(\mathrm{z}, \mathrm{z}) \\
+\mathrm{d}(\mathrm{z}, \mathrm{z}) \mathrm{d}(\mathrm{Cz}, \mathrm{z})
\end{array}\right] \\
& +\alpha_{2}\left[\begin{array}{l}
\mathrm{d}(\mathrm{Cz}, \mathrm{z}) \mathrm{d}(\mathrm{CDz}, \mathrm{z}) \\
+\mathrm{d}(\mathrm{z}, \mathrm{z}) \mathrm{d}(\mathrm{z}, \mathrm{z})
\end{array}\right] \\
{[\mathrm{d}(\mathrm{Cz}, \mathrm{z})]^{2} \leq } & \alpha_{2}[\mathrm{~d}(\mathrm{Cz}, \mathrm{z}) \mathrm{d}(\mathrm{Cz}, \mathrm{z})]
\end{aligned}
$$

Which is a contradiction. Hence

$$
\mathrm{Cz}=\mathrm{z} .
$$

Now since $\mathrm{Cz}=\mathrm{z}$, by condition (1) $\mathrm{z} \in \mathrm{DPV}(\mathrm{X})$. Also DPV is self map of $X$, so there exists a point $u \in X$ such that

$$
\mathrm{z}=\mathrm{Cz}=\mathrm{DPVu}
$$

Moreover by putting $\mathrm{Cz}=\mathrm{z}$ and $\mathrm{x}_{2 \mathrm{n}-1}=\mathrm{u}$ in condition (3.2), we obtain

$$
\begin{gathered}
{[\mathrm{d}(\mathrm{z}, \text { Eu })]^{2} \leq \alpha_{1}\left[\begin{array}{l}
\mathrm{d}(\mathrm{z}, \text { FKMz }) \mathrm{d}(\text { Eu, DPVu }) \\
+\mathrm{d}(\text { Eu, FKMz }) \mathrm{d}(\mathrm{z}, \text { DPVu })
\end{array}\right]} \\
+\alpha_{2}\left[\begin{array}{l}
\mathrm{d}(\mathrm{z}, \text { FKMz }) \mathrm{d}(\mathrm{z}, \text { DPVu }) \\
+\mathrm{d}(\text { Eu, DPVu }) \mathrm{d}(\text { Eu, FKMz })
\end{array}\right] \\
{[\mathrm{d}(\mathrm{z}, \mathrm{Eu})]^{2} \leq \alpha_{1}[\mathrm{~d}(\mathrm{z}, \mathrm{z}) \mathrm{d}(\text { Eu, z })+\mathrm{d}(\text { Eu, z }) \mathrm{d}(\mathrm{z}, \mathrm{z})]} \\
+\alpha_{2}[\mathrm{~d}(\mathrm{z}, \mathrm{z}) \mathrm{d}(\mathrm{z}, \mathrm{z})+\mathrm{d}(\text { Eu, z }) \mathrm{d}(\text { Eu, z })] \\
{[\mathrm{d}(\mathrm{z}, \text { Eu })]^{2} \leq \alpha_{2}[\mathrm{~d}(\mathrm{z}, \mathrm{Eu})]^{2}}
\end{gathered}
$$

Which is a contradiction.

Hence $\mathrm{Eu}=\mathrm{z}$, i.e., $\mathrm{z}=\mathrm{DPVu}=\mathrm{Eu}$.

By condition (5), we have

$$
[\mathrm{d}(\mathrm{DPV}(\mathrm{Eu}), \mathrm{E}(\mathrm{DPVu}))]=0 .
$$

Hence d(DPVz, Ez $)=0$ i.e., DPVz = Ez.

Now

$$
\begin{aligned}
& {[\mathrm{d}(\mathrm{z}, \mathrm{DPVz})]^{2}=[\mathrm{d}(\mathrm{Cz}, \mathrm{Ez})]^{2}} \\
& \leq \alpha_{1}\left[\begin{array}{l}
\mathrm{d}(\mathrm{Cz}, \text { FKMz }) \mathrm{d}(\text { Ez, DPVz }) \\
+\mathrm{d}(\text { Ez, FKMz }) \mathrm{d}(\mathrm{Cz}, \mathrm{DPVz})
\end{array}\right] \\
& +\alpha_{2}\left[\begin{array}{l}
\mathrm{d}(\mathrm{Cz}, \text { FKMz }) \mathrm{d}(\mathrm{Cz}, \mathrm{DPVz}) \\
+\mathrm{d}(\text { Ez, DPVz }) \mathrm{d}(\text { Ez, FKMz })
\end{array}\right] \\
& {[\mathrm{d}(\mathrm{z}, \mathrm{DPVz})]^{2} \leq \alpha_{1}\left[\begin{array}{l}
\mathrm{d}(\mathrm{z}, \mathrm{z}) \mathrm{d}(\mathrm{DPVz}, \mathrm{DPVz}) \\
+\mathrm{d}(\text { DPVz, z }) \mathrm{d}(\mathrm{z}, \text { DPVz })
\end{array}\right]} \\
& +\alpha_{2}\left[\begin{array}{l}
\mathrm{d}(\mathrm{z}, \mathrm{z}) \mathrm{d}(\mathrm{z}, \mathrm{DPVz}) \\
+\mathrm{d}(\text { DPVz, DPVz }) \mathrm{d}(\text { DPVz, z })
\end{array}\right] \\
& {[\mathrm{d}(\mathrm{z}, \mathrm{DPVz})]^{2} \leq \alpha_{1}[\mathrm{~d}(\mathrm{DPVz}, \mathrm{z}) \mathrm{d}(\mathrm{z}, \mathrm{DPVz})]} \\
& {[\mathrm{d}(\mathrm{z}, \mathrm{DPVz})]^{2} \leq \alpha_{1}[\mathrm{~d}(\mathrm{z}, \mathrm{DPVz})]^{2}}
\end{aligned}
$$

Which is a contradiction.

$$
\text { Hence } \mathrm{z}=\mathrm{DPVz} \text {, i.e., } \mathrm{z}=\mathrm{DPVz}=\mathrm{Ez} \text {. }
$$

Now to prove $\mathrm{Vz}=\mathrm{z}$, put $\mathrm{x}=\mathrm{z}$ and $\mathrm{y}=\mathrm{Vz}$ in (1) and using (3.1), (3.3) and (3.5), we have

$$
\begin{aligned}
& {[\mathrm{d}(\mathrm{Cz}, \mathrm{E}(\mathrm{Vz}))]^{2}} \\
& \leq \alpha_{1}\left[\begin{array}{l}
\mathrm{d}(\mathrm{Cz}, \mathrm{FKM}(\mathrm{Vz})) \mathrm{d}(\mathrm{E}(\mathrm{Vz}), \operatorname{DPV}(\mathrm{Vz})) \\
+\mathrm{d}(\mathrm{E}(\mathrm{Vz}), \text { FKMz }) \mathrm{d}(\mathrm{Cz}, \operatorname{DPV}(\mathrm{Vz}))
\end{array}\right] \\
& +\alpha_{2}\left[\begin{array}{l}
\mathrm{d}(\mathrm{Cz}, \mathrm{FKMz}) \mathrm{d}(\mathrm{Cz}, \mathrm{DPV}(\mathrm{Vz})) \\
+\mathrm{d}(\mathrm{E}(\mathrm{Vz}), \mathrm{DPV}(\mathrm{Vz})) \mathrm{d}(\mathrm{E}(\mathrm{Vz}), \text { FKMz })
\end{array}\right] \\
& {[\mathrm{d}(\mathrm{z}, \mathrm{Vz})]^{2}} \\
& \leq \alpha_{1}[\mathrm{~d}(\mathrm{z}, \mathrm{Vz}) \mathrm{d}(\mathrm{Vz}, \mathrm{Vz})+\mathrm{d}(\mathrm{Vz}, \mathrm{z}) \mathrm{d}(\mathrm{z}, \mathrm{Vz})] \\
& +\alpha_{2}[d(z, z) d(z, V z)+d(V z, V z) d(V z, z)] \\
& {[\mathrm{d}(\mathrm{z}, \mathrm{Vz})]^{2} \leq \alpha_{1}[\mathrm{~d}(\mathrm{z}, \mathrm{Vz})]^{2}}
\end{aligned}
$$


Which is a contradiction.

Hence $\mathrm{z}=\mathrm{Vz}$. Since $\mathrm{DPVz}=\mathrm{z}$, implies that $\mathrm{DPz}=\mathrm{z}$.

Now to prove $\mathrm{Pz}=\mathrm{z}$, put $\mathrm{x}=\mathrm{z}$ and $\mathrm{y}=\mathrm{Pz}$ in (1) and using (3.1), (3.3) and (3.5), we have

$$
\begin{aligned}
& {[\mathrm{d}(\mathrm{Cz}, \mathrm{E}(\mathrm{Pz}))]^{2}} \\
& \leq \alpha_{1}\left[\begin{array}{l}
\mathrm{d}(\mathrm{Cz}, \mathrm{FKM}(\mathrm{Pz})) \mathrm{d}(\mathrm{E}(\mathrm{Pz}), \mathrm{DPV}(\mathrm{Pz})) \\
+\mathrm{d}(\mathrm{E}(\mathrm{Pz}), \mathrm{FKMz}) \mathrm{d}(\mathrm{Cz}, \mathrm{DPV}(\mathrm{Pz}))
\end{array}\right] \\
& +\alpha_{2}\left[\begin{array}{l}
\mathrm{d}(\mathrm{Cz}, \mathrm{FKMz}) \mathrm{d}(\mathrm{Cz}, \mathrm{DPV}(\mathrm{Pz})) \\
+\mathrm{d}(\mathrm{E}(\mathrm{Pz}), \mathrm{DPV}(\mathrm{Pz})) \mathrm{d}(\mathrm{E}(\mathrm{Pz}), \mathrm{FKMz})
\end{array}\right] \\
& {[\mathrm{d}(\mathrm{z}, \mathrm{Pz})]^{2}} \\
& \leq \alpha_{1}[\mathrm{~d}(\mathrm{z}, \mathrm{Pz}) \mathrm{d}(\mathrm{Pz}, \mathrm{Pz})+\mathrm{d}(\mathrm{Pz}, \mathrm{z}) \mathrm{d}(\mathrm{z}, \mathrm{Pz})] \\
& +\alpha_{2}[\mathrm{~d}(\mathrm{z}, \mathrm{z}) \mathrm{d}(\mathrm{z}, \mathrm{Pz})+\mathrm{d}(\mathrm{Pz}, \mathrm{Pz}) \mathrm{d}(\mathrm{Pz}, \mathrm{z})] \\
& {[\mathrm{d}(\mathrm{z}, \mathrm{Pz})]^{2} \leq \alpha_{1}[\mathrm{~d}(\mathrm{z}, \mathrm{Pz})]^{2}}
\end{aligned}
$$

Which is a contradiction.

Hence $\mathrm{Pz}=\mathrm{z}$. Since $\mathrm{DPz}=\mathrm{z}$, implies that $\mathrm{Dz}=\mathrm{z}$.

Now to prove $\mathrm{Mz}=\mathrm{z}$, put $\mathrm{x}=\mathrm{Mz}$ and $\mathrm{y}=\mathrm{z}$ in (1) and using (3.1), (3.3) and (3.5), we have

$$
\begin{aligned}
& \quad[\mathrm{d}(\mathrm{C}(\mathrm{Mz}), \mathrm{Ez})]^{2} \\
& \leq \alpha_{1}\left[\begin{array}{l}
\mathrm{d}(\mathrm{C}(\mathrm{Mz}), \mathrm{FKM}(\mathrm{Mz})) \mathrm{d}(\mathrm{Ez}, \mathrm{DPVz}) \\
+\mathrm{d}(\mathrm{Ez}, \mathrm{FKM}(\mathrm{Mz})) \mathrm{d}(\mathrm{C}(\mathrm{Mz}), \mathrm{DPVz})
\end{array}\right] \\
& +\alpha_{2}\left[\begin{array}{l}
\mathrm{d}(\mathrm{C}(\mathrm{Mz}), \mathrm{FKM}(\mathrm{Mz})) \mathrm{d}(\mathrm{C}(\mathrm{Mz}), \mathrm{DPVz}) \\
+\mathrm{d}(\mathrm{Ez}, \mathrm{DPVz}) \mathrm{d}(\mathrm{Ez}, \mathrm{FKM}(\mathrm{Mz}))
\end{array}\right] \\
& {\left[\begin{array}{c}
\mathrm{d}(\mathrm{Mz}, \mathrm{z})]^{2} \\
\leq \\
\quad \alpha_{1}[\mathrm{~d}(\mathrm{Mz}, \mathrm{Mz}) \mathrm{d}(\mathrm{z}, \mathrm{z})+\mathrm{d}(\mathrm{z}, \mathrm{Mz}) \mathrm{d}(\mathrm{Mz}, \mathrm{z})]
\end{array}\right.} \\
& \quad+\alpha_{2}[\mathrm{~d}(\mathrm{Mz}, \mathrm{Mz}) \mathrm{d}(\mathrm{Mz}, \mathrm{z})+\mathrm{d}(\mathrm{z}, \mathrm{z}) \mathrm{d}(\mathrm{z}, \mathrm{Mz})] \\
& {[\mathrm{d}(\mathrm{Mz}, \mathrm{z})]^{2} \leq \alpha_{1}[\mathrm{~d}(\mathrm{Mz}, \mathrm{z})]^{2}}
\end{aligned}
$$

Which is a contradiction.

Hence $\mathrm{Mz}=\mathrm{z}$. Since $\mathrm{FKMz}=\mathrm{z}$, implies that $\mathrm{FKz}=\mathrm{z}$.

Now to prove $\mathrm{Kz}=\mathrm{z}$, put $\mathrm{x}=\mathrm{Kz}$ and $\mathrm{y}=\mathrm{z}$ in (1) and using (3.1), (3.3) and (3.5), we have

$$
\begin{aligned}
& \quad[\mathrm{d}(\mathrm{C}(\mathrm{Kz}), \mathrm{Ez})]^{2} \\
& \leq \alpha_{1}\left[\begin{array}{l}
\mathrm{d}(\mathrm{C}(\mathrm{Kz}), \mathrm{FKM}(\mathrm{Kz})) \mathrm{d}(\mathrm{Ez}, \mathrm{DPVz}) \\
+\mathrm{d}(\mathrm{Ez}, \mathrm{FKM}(\mathrm{Kz})) \mathrm{d}(\mathrm{C}(\mathrm{Kz}), \mathrm{DPVz})
\end{array}\right] \\
& +\alpha_{2}\left[\begin{array}{l}
\mathrm{d}(\mathrm{C}(\mathrm{Kz}), \mathrm{FKM}(\mathrm{Kz})) \mathrm{d}(\mathrm{C}(\mathrm{Kz}), \mathrm{DPVz})] \\
+\mathrm{d}(\mathrm{Ez}, \mathrm{DPVz}) \mathrm{d}(\mathrm{Ez}, \mathrm{FKM}(\mathrm{Kz}))
\end{array}\right] \\
& {[\mathrm{d}(\mathrm{Kz}, \mathrm{z})]^{2}} \\
& \leq \alpha_{1}[\mathrm{~d}(\mathrm{Kz}, \mathrm{Kz}) \mathrm{d}(\mathrm{z}, \mathrm{z})+\mathrm{d}(\mathrm{z}, \mathrm{Kz}) \mathrm{d}(\mathrm{Kz}, \mathrm{z})] \\
& +\alpha_{2}[\mathrm{~d}(\mathrm{Kz}, \mathrm{Kz}) \mathrm{d}(\mathrm{Kz}, \mathrm{z})+\mathrm{d}(\mathrm{z}, \mathrm{z}) \mathrm{d}(\mathrm{z}, \mathrm{Kz})] \\
& {[\mathrm{d}(\mathrm{Mz}, \mathrm{z})]^{2} \leq \alpha_{1}[\mathrm{~d}(\mathrm{Kz}, \mathrm{z}) \mathrm{d}(\mathrm{z}, \mathrm{Kz})]} \\
& {[\mathrm{d}(\mathrm{Mz}, \mathrm{z})]^{2} \leq \alpha_{1}[\mathrm{~d}(\mathrm{Kz}, \mathrm{z})]^{2}}
\end{aligned}
$$

Which is a contradiction.

Hence $\mathrm{Kz}=\mathrm{z}$. Since $\mathrm{FKz}=\mathrm{z}$, implies that $\mathrm{Fz}=\mathrm{z}$. Thus $\mathrm{Cz}=\mathrm{Dz}=\mathrm{Ez}=\mathrm{Fz}=\mathrm{Kz}=\mathrm{Mz}=\mathrm{Pz}=\mathrm{Vz}=\mathrm{z}$. Therefore $\mathrm{z}$ is common fixed point of $\mathrm{C}, \mathrm{D}, \mathrm{E}, \mathrm{F}, \mathrm{K}, \mathrm{M}, \mathrm{P}$ and $\mathrm{V}$. Similarly we can prove this any one of C, D, E, F, P and V is continuous.

\section{Uniqueness}

Suppose $\mathrm{w}$ be another common fixed point of C, D, E, F, K. M, P and V. Then we have

$$
\begin{aligned}
& {[\mathrm{d}(\mathrm{z}, \mathrm{w})]^{2}=[\mathrm{d}(\mathrm{Cz}, \mathrm{Ew})]^{2}} \\
& \leq \alpha_{1}\left[\begin{array}{l}
\mathrm{d}(\mathrm{Cz}, \text { FKMz }) \mathrm{d}(\text { Ew, DPVw }) \\
+\mathrm{d}(\text { Ew, FKMz }) \mathrm{d}(\mathrm{Cz}, \text { DPVw })
\end{array}\right] \\
& +\alpha_{2}\left[\begin{array}{l}
\mathrm{d}(\mathrm{Cz}, \text { FKMz }) \mathrm{d}(\mathrm{Cz}, \text { DPVw }) \\
+\mathrm{d}(\text { Ew, DPVw }) \mathrm{d}(\text { Ew, FKMz })
\end{array}\right] \\
& {[\mathrm{d}(\mathrm{z}, \mathrm{w})]^{2}} \\
& \leq \alpha_{1}[\mathrm{~d}(\mathrm{z}, \mathrm{z}) \mathrm{d}(\mathrm{w}, \mathrm{w})+\mathrm{d}(\mathrm{w}, \mathrm{z}) \mathrm{d}(\mathrm{z}, \mathrm{w})] \\
& +\alpha_{2}[\mathrm{~d}(\mathrm{z}, \mathrm{z}) \mathrm{d}(\mathrm{z}, \mathrm{w})+\mathrm{d}(\mathrm{w}, \mathrm{w}) \mathrm{d}(\mathrm{w}, \mathrm{z})] \\
& {[\mathrm{d}(\mathrm{z}, \mathrm{w})]^{2} \leq \alpha_{1}[\mathrm{~d}(\mathrm{z}, \mathrm{w}) \mathrm{d}(\mathrm{z}, \mathrm{w})]} \\
& {[\mathrm{d}(\mathrm{z}, \mathrm{w})]^{2} \leq \alpha_{1}[\mathrm{~d}(\mathrm{z}, \mathrm{w})]^{2}}
\end{aligned}
$$

Which is a contradiction.

Hence $\mathrm{z}=\mathrm{w}$. Therefore $\mathrm{z}$ is a unique common fixed point of C, D, E, F, K, M, P and V.

Corollary: Let C, D, E, K, M and V be self maps of a complete metric space $(\mathrm{X}, \mathrm{d})$ satisfying the following conditions:

(1) $\mathrm{C}(\mathrm{X}) \subseteq \mathrm{DV}(\mathrm{X})$ and $\mathrm{E}(\mathrm{X}) \subseteq \mathrm{KM}(\mathrm{X})$

$$
\begin{gathered}
{[\mathrm{d}(\mathrm{Cx}, \text { Ey })]^{2}} \\
(2) \leq \alpha_{1}\left[\begin{array}{l}
\mathrm{d}(\mathrm{Cx}, \text { KMx }) \mathrm{d}(\text { Ey, DPVy }) \\
+\mathrm{d}(\text { Ey, KMx }) \mathrm{d}(\mathrm{Cx}, \text { DVy })
\end{array}\right] \\
+\alpha_{2}\left[\begin{array}{l}
\mathrm{d}(\mathrm{Cx}, \text { KMx }) \mathrm{d}(\mathrm{Cx}, \text { DVy }) \\
+\mathrm{d}(\text { Ey, Vy }) \mathrm{d}(\text { Ey, KMx })
\end{array}\right]
\end{gathered}
$$

Where $0 \leq \alpha_{1}+2 \alpha_{2}<1 ; \propto_{1}, \propto_{2} \geq 0$.

(3) Let $x_{0} \in X$ then by (1) there exists $x_{1} \in X$ such that $\mathrm{DVx}_{1}=\mathrm{Cx}_{0}$ and for $\mathrm{x}_{1}$ there exists $\mathrm{x}_{2} \in \mathrm{X}$ such that $\mathrm{KMx}_{2}=\mathrm{Ex}_{1}$ and so on.continuing this process we candefine a sequence $\left\{y_{n}\right\}$ in $X$ such that

$$
\begin{aligned}
& \mathrm{y}_{2 \mathrm{n}+1}=\mathrm{DVx}_{2 \mathrm{n}+1}=\mathrm{Cx}_{2 \mathrm{n}} \\
& \text { and } \mathrm{y}_{2 \mathrm{n}}=\mathrm{KMx}_{2 \mathrm{n}}=\mathrm{Ex}_{2 \mathrm{n}-1} .
\end{aligned}
$$

Then the sequence $\left\{\mathrm{y}_{\mathrm{n}}\right\}$ is a Cauchy sequence in $\mathrm{X}$.

(4) One of C, E, KM, DV is continuous.

(5) $[C, K M]$ and $[E, D V]$ are compatible of type (R) on X.

Then $\mathrm{C}, \mathrm{D}, \mathrm{E}, \mathrm{K}, \mathrm{M}$ and $\mathrm{V}$ have a unique common fixed point in $\mathrm{X}$.

\section{Conclusion}

In this paper, we have presented common fixed point theorem for eight mappings in metric spaces through concept of compatibility. 


\section{Acknowledgement}

The Authors are thankful to the anonymous referees for his valuable suggestions for the improvement of this paper.

\section{References}

[1] Singh, B. and Chauhan, M.S., "On common fixed points of four mappings”, Bull. Cal. Math. Soc., 88, 451-456, 1996.
[2] Jungck, G. "Compatible maps and common fixed points", Inter. J. Math. And Math. Sci., 9, 771-779, 1986.

[3] Pathak, H.K., Chang, S.S. and Cho, Y.J., "Fixed point theorem for compatible mappings of type (P)”, Indian J. Math. 36(2), 151-166, 1994.

[4] Koireng Meitei, M., Ningombam, L. and Rohen, Y. "common fixed point theorem of compatible mappings of type(R)", Gen.Math. Notes, 10(1), 58-62, May 2012.

[5] Rohen, Y., Singh, M.R. and Shambhu, L., "Common fixed points of compatible mapping of type (C) in Banach Spaces", Proc. of Math.Soc., 11(2), 42-50, 2011. 